\title{
Tumor staging using 3.0 T multiparametric MRI in prostate cancer: impact on treatment decisions for radical radiotherapy
}

\author{
Felipe Couñago ${ }^{1 *}$, Elia del Cerro', Ana Aurora Díaz-Gavela' ${ }^{1}$, Francisco José Marcos ${ }^{1}$, Manuel Recio², \\ David Sanz-Rosa ${ }^{3}$, Israel Thuissard ${ }^{4}$, Karmele Olacireguii $^{5}$, María Mateo ${ }^{6}$ and Laura Cerezo ${ }^{7}$
}

\begin{abstract}
To assess and validate the incorporation of the multiparametric magnetic resonance imaging (mpMRI) tumour category (mT-category) to the conventional clinical tumour category (cT-category), in order to guide the radiotherapy (RT) treatment decisions in prostate cancer. In addition, to identify the clinical factors associated to the technique reliability. mpMRI was performed in 274 prostate cancer patients in order to refine the treatment decisions according to PSA, Gleason Score (GS) and cT-category. Comparisons between the cT and mT-category were performed, as well as the impact on the RT treatment [target volume, doses and hormonal therapy $(\mathrm{HT})$ ] independently if it was finally performed. Changes in $\mathrm{HT}$ indication for intermediate risk were also analyzed. mpMRI validation was performed with pathological staging ( $n=90$ patients finally decided to join surgery). The mpMRl upstaging range was $86-94 \%$ for any PSA value or GS. Following mpMRI, $32.8 \%$ of the patients (90/274) were assigned to a different risk group. Compared to cT-category, mpMRI identified more intermediate-risk (46.4 vs. $59.5 \%$ ) and high-risk (19.0 vs. $28.8 \%$ ) prostate cancer patients. This resulted in a higher indication $(p<0.05)$ of seminal vesicle irradiation $(63.5$ vs. $70.0 \%)$, inclusion of any extracapsular disease (T3-T4) within the target volume (1.8 vs. $18.2 \%$ ), higher doses (65.3 vs. $88.3 \%$ ) and HT associated to RT (45.6 vs. 62.4\%). Global accuracy for mpMRI was higher compared to DRE/TRUS (8.9 vs. $71.1 \%$, $p<0.05)$. mpMRI reliability was independent of PSA or GS. mpMRI tumor staging significantly modified the RT treatment decisions in all prostate cancer risk groups.
\end{abstract}

Keywords: Prostate cancer, Staging, Radiotherapy, Multiparametric MRI

\section{Background}

Clinical tumoural category (cT-category) is a determining factor in prostate cancer management. Thus, cT-category, along with prostate-specific antigen level (PSA) and Gleason score (GS), contribute to establishing tumour recurrence risk groups, and subsequently to making radiotherapy treatment (RT) decisions [such as target volume, total dose, and addition of hormonal therapy (HT)]. In determining the cT-category in prostate cancer, digital rectal examination (DRE) and transrectal ultrasound

\footnotetext{
*Correspondence: fcounago@gmail.com

${ }^{1}$ Department of Radiation Oncology, Hospital Universitario Quiron Madrid, Calle Diego de Velazquez, 1, Pozuelo de Alarcón, 28223 Madrid, Spain

Full list of author information is available at the end of the article
}

(TRUS) remain the gold-standard methods recommended in almost all guidelines and protocols (Mohler et al. 2014; Heidenreich et al. 2014). However, both tests have very low reliability and high interobserver variability (Philip et al. 2005; Smith and Catalona 1995). This could result in erroneous estimation of the cT-category, potentially resulting in inappropriate treatment, particularly in relation to RT.

In recent years, multiparametric magnetic resonance imaging (mpMRI) has been the most reliable technique for local tumour staging in prostate cancer patients, compared with conventional tests such as DRE, TRUS and morphological MRI, as well as predictive tools such as the Partin tables and the Kattan nomogram (Hricak et al. 1987; Mullerad et al. 2005; Augustin et al. 2009; Wang et al. 2007). However, few studies have focused on the

\section{黛 Springer}


impact of mpMRI staging in relation to RT target volumes, total dose administered, or the use of HT (Couñago et al. 2014; Panje et al. 2015).

Although heterogeneity is common among institutional protocols (Horsley et al. 2015), at our institution, the recommendation for low-risk patients is to treat the prostate with RT (76-78 Gy) without associated HT; by contrast, for organ-confined (T1-T2) intermediate-risk and high-risk patients, the irradiation of both prostate and seminal vesicles (78-80 Gy) is indicated (Mohler et al. 2014; Heidenreich et al. 2014). In high-risk patients with locally advanced disease (T3-T4), it is also necessary to extend the target volume beyond the prostatic capsule to encompass any type of extracapsular extension (ECE) (Boehmer et al. 2006; Hayden et al. 2010). Long-term HT treatment $(\geq 24 \mathrm{~m})$ is recommended for high-risk patients. Short-term treatment $(4-6 \mathrm{~m})$, while controversial, is recommended for intermediate-risk patients with unfavourable prognostic factors (Zapatero et al. 2015; D'Amico 2015). Either prophylactic lymph node irradiation or brachytherapy boost following RT are optional in intermediate-risk and high-risk patients (Mohler et al. 2014; Heidenreich et al. 2014).

With this in mind, the principal aim of our study was to analyse and validate the consequences of mpMRI staging for RT treatment decisions (target volume, doses and HT) in prostate cancer, as well as to determine the clinical factors associated with mpMRI reliability.

\section{Methods}

\section{Study design}

This is a retrospective study conducted in our Oncology Department with the approval of Quiron Hospital's Ethics Committee. Between January 2009 and April 2015, 274 patients with histological diagnoses of primary prostate cancer, mpMRI-categorized and considered for definitive treatment with RT \pm HT or radical prostatectomy (RP), were included in our study. Patients without mpMRI staging prior to any treatment $(n=175)$ or with non-assessed mpMRI due to bleeding after prostatic biopsy $(n=6)$ were excluded. Ten patients without cTcategory were also excluded. All patients were assessed by Urology and Radiation Oncology Services before the definitive treatment decision. The completion of RT treatment was not essential for inclusion in the study, as decisions regarding target volume, doses, and HT depend exclusively on tumour category, PSA value and GS.

Before treatment was started, patients' baseline characteristics were evaluated, including age at diagnosis, DRE, PSA value at diagnosis, and a TRUS-guided biopsy from which the patients' GS were obtained. Other imaging techniques (CT and bone scan) were optional, according to international guidelines (Mohler et al. 2014;
Heidenreich et al. 2014) and the physician's judgment, basically in intermediate and high risk patients. cT-category before mpMRI was assigned on the basis of DRE and TRUS. Tumour category was assessed according to the American Joint Committee on Cancer (AJCC) system, 7th edition (AJCC 2010). Based on PSA values, GS and cT-category, relapse risk groups were established following the National Comprehensive Cancer Network guidelines version 2.2014: low-risk, $\leq \mathrm{T} 2 \mathrm{a}, \mathrm{GS} \leq 6$ and pretreatment PSA $<10 \mathrm{ng} / \mathrm{mL}$; intermediate-risk, T2b-T2c or GS $=7$ or PSA $10-20 \mathrm{ng} / \mathrm{mL}$; high-risk, T3-T4 or GS $>7$ or PSA $>20 \mathrm{ng} / \mathrm{mL}$; metastatic, $\mathrm{N}+$ or $\mathrm{M}+$ (Mohler et al. 2014).

Based on the prostate cancer clinical protocol in force in our institution's radiotherapy unit, initial indication (target volume, doses and HT) was established according to cT-category; ultimate indications were based on MRI tumour category (mT-category). Up- or downstaging, changes in risk group, and alterations in RT treatment due to tumour category variations were analysed. In addition, for patients whose final treatment consisted of $\mathrm{RP}(\mathrm{n}=90)$, the mT-category was compared to the pathologic tumour category (pT-category), which allowed us to validate mpMRI results.

\section{mpMRI technique}

mpMRI was performed on an eight-channel torso-array antenna 3 Tesla (General Electric, USA) in previously prepared patients. The study protocol was extensively described in a previous study (Couñago et al. 2014). In brief, morphological imaging included T1- and T2-weighted sequences and functional studies included diffusion-weighted imaging (DWI) and dynamic contrast-enhanced (DCE) imaging. The apparent diffusion coefficient (ADC) map was calculated while using DWI. DCE imaging was performed applying contrast with gadolinium. In the DCE study, time-intensity curves were calculated. The mpMRI was performed after conventional clinical staging, and always interpreted by one uroradiologist experienced in pelvic MRI.

\section{Risk-adapted RT}

Patients underwent treatment in a multienergetic lineal accelerator with intensity modulated radiotherapy (IMRT). In low-risk patients, the dose was 76-78 Gy, increasing to 78-80 Gy in intermediate-risk and highrisk patients. The clinical target volume (CTV) included only the prostate in low-risk patients, while the prostate and seminal vesicles were included in intermediate-risk and high-risk patients. CTV was extended to include ECE in T3-T4 patients, following the recommendations of clinical guidelines (Boehmer et al. 2006; Hayden et al. 2010). The pelvis was not included, except in cases 
with clinical pelvic node involvement. High-risk patients received long-term HT (2-3 years) and intermediate-risk patients with unfavourable factors received short-term HT (4-6 months). Until 2014, the unfavourable factors according to the initial criteria were a GS of $7(4+3)$, or three unfavourable intermediate risk factors (T2b + PSA 10-20 ng/mL + GS $3+4$ ), or T2c by DRE/TRUS; more recently, unfavourable risk factors have been established according to memorial sloan kettering cancer center (MSKCC) criteria: GS $4+3$, or at least two intermediaterisk factors, or at least one intermediate-risk factor and a positive prostate biopsies (ppb) percentage greater than 50 \% (D’Amico 2015; Zumsteg et al. 2013).

\section{Statistical analysis}

The data analysis was performed with IBM SPSS statistics version 21.0 (IBM Corp; USA). Descriptive statistics are reported as median (IQR, interquartile range) or mean (SD, Standard Deviation) for continuous variables, and as absolute or relative frequencies for categorical variables. Patient basal characteristics and the differences in treatment impact on DRE/TRUS vs. mpMRI, as well as the reliability of the diagnostic tests, were compared using the Chi square test and Fisher's exact test, both for categorical variables. The U Mann-Whitney test was used for the non-parametric quantitative variables. To analyse the relationship between the reliability of mpMRI and the clinical variables, a univariate and multivariate logistic regression analysis was performed.

\section{Results}

The baseline patient characteristics of the entire cohort $(\mathrm{n}=274)$ and the surgical patient cohort $(\mathrm{n}=90)$ are summarised in Table 1 . Upstaging occurred in $90.9 \%$ of the patients (249/274 patients; see Fig. 1). The upstaging range was $86.1-94.2 \%$ for any PSA or GS value. Downstaging was not observed. Following mpMRI, $32.8 \%$ of the patients $(90 / 274)$ were assigned to a different risk group. Finally, decisions concerning RT were changed in $43.8 \%$ (initial criteria) or $52.5 \%$ (MSKCC criteria) of the patients, depending on the criteria applied to indicate HT in intermediate-risk patients.

Upstaging from cT1-T2a to mT2b-T2c occurred in 137 patients of a total of 239 patients (57.3\%). 58 out of 239 patients (24.2\%) upward shift from low-risk to intermediate-risk resulting in a modification of target volume due to the inclusion of seminal vesicles, and the increase in dosage from 76-78 to 78-80 Gy. In 7 of a total of 11 of the intermediate-risk patients (cT1-cT2a and GS $3+4$ and PSA $10-20 \mathrm{ng} / \mathrm{mL}$ ), short-term HT was also indicated, as 3 unfavorable risk factors were present. However, if the most recent MSKCC criteria had been applied, 31 out of 41 intermediate-risk patients [cT1-cT2a and
Table 1 Patients characteristics

\begin{tabular}{lll}
\hline Characteristics & Total cohort (\%) & $\begin{array}{l}\text { Radical } \\
\text { prostatectomy (\%) }\end{array}$ \\
\hline Patients & $274(100)$ & $90(100)$ \\
Age (years) & $66.8 \pm 8.1$ & $60.9 \pm 6.8$ \\
PSA (ng/mL) & $179(65.3)$ & $68(75.6)$ \\
$<10$ & $66(24.1)$ & $18(20.0)$ \\
$10-20$ & $29(10.6)$ & $4(4.4)$ \\
$>20$ & & \\
Gleason score & $137(50.0)$ & $60(66.7)$ \\
$\leq 6$ & $108(39.4)$ & $24(26.7)$ \\
7 & $29(10,6)$ & $6(6.6)$ \\
$\geq 8$ & & \\
CT-category before mpMRI & $239(87.2)$ & $84(93.3)$ \\
$\leq$ T2a & $30(11.0)$ & $6(6.7)$ \\
T2b-T2c & $5(1.8)$ & 0 \\
T3a & 0 & 0 \\
T3b & 0 & 0 \\
T4 & & $16(17.8)$ \\
Risk groups & $95(34.7)$ & $43(47.8)$ \\
Low & $127(46.4)$ & $37(41.1)$ \\
Intermediate & $52(19.0)$ & $10(11.1)$ \\
High & & $49(54.4)$ \\
Positive prostate biopsies & $158(57.7)$ & \\
$<50 \%$ & $65(23.7)$ & \\
$\geq 50 \%$ & $51(18.6)$ & \\
(Non classified) & & \\
\hline
\end{tabular}

$m p M R I$ multiparametric magnetic resonance imaging, PSA prostate-specific antigen

(GS $3+4$ or PSA $10-20 \mathrm{ng} / \mathrm{mL}$ or $\mathrm{ppb}>50 \%$ )], would have received short-term HT.

Among the 269 cT1-T2 patients, 45 (16.7 \%) progressed to $\mathrm{mT} 3-\mathrm{T} 4$, based on the detection of any type of ECE in mpMRI. This entailed an alteration of target volume due to the inclusion of seminal vesicles, as well as ECE, an increase in dosage, and inclusion of long-term HT for five low-risk patients. Of these 45 patients, 22 were allocated to the intermediate-risk group, resulting in the modification of the target volume due to the inclusion of ECE and the addition of long-term HT. Lastly, the inclusion of ECE in the target volume was the only alteration in 18 high-risk patients.

Of a total of five cT3a patients, one progressed to mT3b, but this case did not imply modifications of final RT treatment decisions, as in high-risk patients seminal vesicles are always irradiated at full doses according to our institution's protocol. Of the remaining four cT3a patients, three progressed to $\mathrm{mT} 4$ and consequently were treated with an increase in target volume to enable infiltration of the rectum or the bladder. Lastly, out of 127 


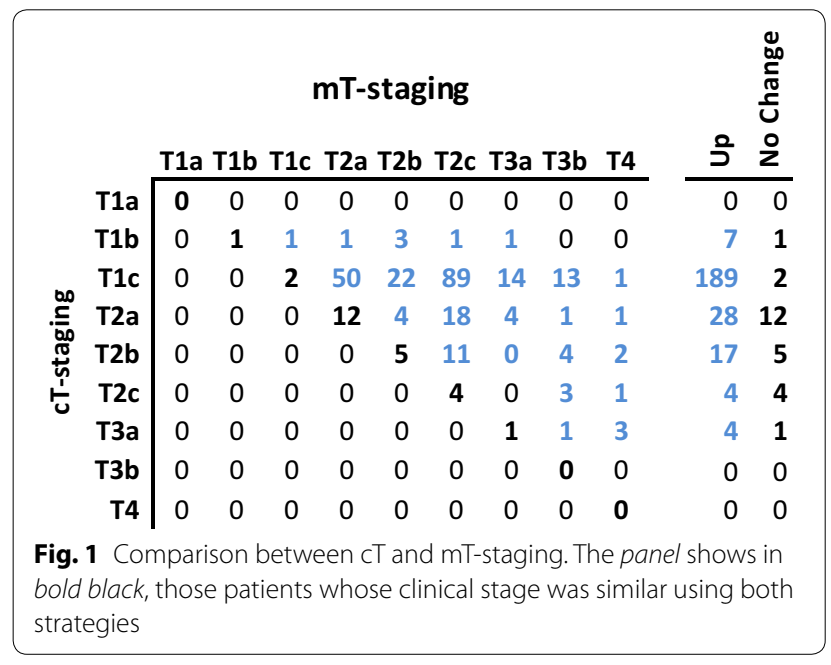

intermediate-risk and 52 high-risk patients, stage IV was diagnosed in two and four patients respectively (3.4\%). In these patients, treatment consisted of either HT exclusively, or pelvic RT and long-term HT.

In general, the mpMRI staging led to the identification of more intermediate-risk and high-risk patients compared to conventional clinical staging. As a result, a higher occurrence of irradiation of seminal vesicles, inclusion of any ECE (T3-T4), and more frequent prescription of combined RT/HT, as well as higher doses of RT ( $<<0.05)$, were observed (Table 2 ).

In order to validate the mpMRI results, 90 patients treated with RP were analysed. The mT-category and the pT-category were compared (Table 3). Global accuracy of cT-staging with DRE/TRUS was $8.9 \%$ (8/90), while it was $71.1 \%(64 / 90)$ for mpMRI. Among the 51 patients upstaged from cT1-T2a to mT2b-T2c, 38 patients $(74.5 \%)$ showed MRI findings in agreement with the pathological staging. For the 12 patients upstaged from cT1-T2 to $\mathrm{mT} 3$, this agreement occurred in seven patients (58.3\%). This entailed a $91.1 \%$ accuracy, $70.0 \%$ (95\% CI 41.6-98.4) sensitivity, 93.8 \% (95 \% CI 88.599.1) specificity, $58.3 \%$ positive predictive value (95\% CI $30.4-86.2$ ) and a $96.2 \%$ negative predictive value (95\% CI 91.9-100) in the MRI staging of ECE (T3) patients.

Having the pT-staging as the gold standard, there was less disagreement in the RT treatment decisions (CTV, HT indication, RT prescription dose) with mT-staging compared to c'T-staging (Table 3).

\section{Clinical factors associated to mpMRI reliability}

Possible clinical variables associated with an increase in mpMRI reliability were analysed in relation to tumour staging through univariate and multivariate logistic regression analysis. None of the clinical factors analysed
Table 2 Impact of mpMRI tumor staging on RT treatment prescription (target volume, doses and hormonal therapy)

\begin{tabular}{|c|c|c|c|}
\hline Characteristics & DRE/TRUS n (\%) & mpMRI n (\%) & $p$ value \\
\hline \multicolumn{4}{|l|}{ T-category } \\
\hline $\mathrm{T} 1-\mathrm{T} 2 \mathrm{a}$ & $239(87.2)$ & $67(24.5)$ & \multirow[t]{3}{*}{$<0.001$} \\
\hline $\mathrm{T} 2 \mathrm{~b}-\mathrm{T} 2 \mathrm{c}$ & $30(11.0)$ & $157(57.3)$ & \\
\hline T3-T4 & $5(1.8)$ & $50(18.2)$ & \\
\hline \multicolumn{4}{|l|}{ Risk group } \\
\hline Low-risk (LR) & $95(34.7)$ & $32(11.7)$ & \multirow[t]{3}{*}{$<0.001$} \\
\hline Intermediate-risk (IR) & $127(46.4)$ & $163(59.5)$ & \\
\hline High-risk (HR) & $52(19.0)$ & $79(28.8)$ & \\
\hline \multicolumn{4}{|l|}{ Target volume } \\
\hline WSS prophylactic & $174(63.5)$ & $192(70.1)$ & \multirow[t]{2}{*}{$<0.001$} \\
\hline $\mathrm{T} 3 \mathrm{a}+\mathrm{T} 3 \mathrm{~b}+\mathrm{T} 4$ & $5(1.8)$ & $50(18.2)$ & \\
\hline \multicolumn{4}{|l|}{ Doses } \\
\hline Low & $95(34.7)$ & $32(11.7)$ & \multirow[t]{2}{*}{$<0.001$} \\
\hline High & $179(65.3)$ & $242(88.3)$ & \\
\hline \multicolumn{4}{|l|}{ Hormonal therapy } \\
\hline \multicolumn{4}{|c|}{ HR and IR (Initial criteria) ${ }^{a}$} \\
\hline No & $182(66.4)$ & $158(57.7)$ & \multirow[t]{2}{*}{0.035} \\
\hline Yes & $92(33.6)$ & $116(42.3)$ & \\
\hline \multicolumn{4}{|c|}{ HR and IR (MSKCC criteria) $)^{b}$} \\
\hline No & $149(54.4)$ & $103(37.6)$ & \multirow[t]{2}{*}{$<0.001$} \\
\hline Yes & $125(45.6)$ & $171(62.4)$ & \\
\hline \multicolumn{4}{|c|}{ Hormonal therapy in IR patients } \\
\hline \multicolumn{4}{|c|}{ Initial criteria } \\
\hline No & $220(80.3)$ & $221(80.7)$ & \multirow[t]{2}{*}{0.914} \\
\hline Yes & $54(19.7)$ & $53(19.3)$ & \\
\hline \multicolumn{3}{|l|}{ MSKCC criteria } & \multirow[t]{3}{*}{0.002} \\
\hline No & $180(65.7)$ & $144(52.6)$ & \\
\hline Yes & $94(34.3)$ & $130(47.4)$ & \\
\hline \multicolumn{4}{|c|}{ Hormonal therapy in HR patients } \\
\hline No & $222(81.0)$ & $195(71.2)$ & \multirow[t]{2}{*}{0.007} \\
\hline Yes & $52(19.0)$ & $79(28.8)$ & \\
\hline
\end{tabular}

WVS seminal vesicles, DRE/TRUS digital rectal exam/transrectal ultrasound a Initial criteria: GS of $7(4+3)$, or three unfavourable IR factors (T2b + PSA $10-20 \mathrm{ng} / \mathrm{mL}+\mathrm{GS} 3+4$ ), or T2c by DRE/TRUS

b MSKCC criteria: GS $4+3$, or at least two IR factors, or at least one IR factor and a positive prostate biopsy (ppb) percentage greater than $50 \%$

(PSA value, GS, \% ppb and age) showed a significant association with mpMRI reliability (Table 4).

\section{Discussion}

We believe that this is the largest cohort of patients in which the impact of mpMRI staging on RT treatment has been analysed. In brief, $90.9 \%$ of patients showed tumour upstaging as a result of mpMRI. Nevertheless, when considering all the risk factors together, such as PSA levels, GS, and tumour category, a change in the risk groups occurred in only $32.8 \%$ of patients. In our 
Table 3 mT-staging validation for the indication of RT treatment

\begin{tabular}{|c|c|c|c|c|}
\hline & DRE/TRUS n (\%) & mpMRI n (\%) & $p$ value & Prostatectomy piece $\mathrm{n}(\%)$ \\
\hline \multicolumn{5}{|l|}{ T-category } \\
\hline $\mathrm{T} 1-\mathrm{T} 2 \mathrm{a}$ & $84(93.7)$ & $24(26.7)$ & $<0.001$ & $23(25.6)$ \\
\hline $\mathrm{T} 2 \mathrm{~b}-\mathrm{T} 2 \mathrm{c}$ & $6(6.7)$ & $54(60.0)$ & & $57(63.3)$ \\
\hline T3-T4 & 0 & $12(13.3)$ & & $10(11.1)$ \\
\hline \multicolumn{5}{|l|}{ Riskgroup } \\
\hline Low-risk & $43(47.8)$ & $13(14.4)$ & $<0.001$ & $10(11.1)$ \\
\hline Intermediate-risk & $37(41.1)$ & $59(65.6)$ & & $65(72.2)$ \\
\hline High-risk & $10(11.1)$ & $18(20.0)$ & & $15(16.7)$ \\
\hline Global reliability for T-category & $8(8.8)$ & $64(71.1)$ & $<0.001$ & \\
\hline Risk group change due to pathological analysis & $37(41.1)$ & $15(16.7)$ & $<0.001$ & \\
\hline \multicolumn{5}{|l|}{ Changes related to RT parameters (HT, Doses, CTV) } \\
\hline Initial criteria & $45(50.0)$ & $16(17.8)$ & $<0.001$ & \\
\hline MSKCC criteria & $59(65.6)$ & $18(20.0)$ & & \\
\hline
\end{tabular}

For patients whose final treatment consisted of RP $(n=90)$, the cT-and mT-category were compared to the pathologic tumour stage (pT-category), which allowed us to validate DRE/TRUS and mpMRI results. Furthermore, the analysis and comparisons related to RT treatment decisions were done for DRE/TRUS vs. mpMRI

$R T$ radiotherapy, $H T$ hormonal therapy, CTV clinical target volume

Table 4 Multivariate analysis of clinical factors associated to $\mathrm{mpMRI}$ reliability

\begin{tabular}{llll}
\hline & OR & $\mathbf{9 5} \% \mathbf{C l}$ & p-value \\
\hline PSA $<10$ & Reference & & \\
PSA $10-20$ & 0.43 & $(0.03-5.05)$ & 0.50 \\
PSA $>20$ & 0.61 & $(0.04-8.21)$ & 0.70 \\
Gleason $\leq 6^{\text {a }}$ & Reference & & \\
Gleason $\geq 7$ & 1.39 & $(0.41-4.65)$ & 0.58 \\
ppb $<50 \%$ & Reference & & \\
ppb $\geq 50 \%$ & 1.07 & $(0.31-3.69)$ & 0.90 \\
Age & 0.92 & $(0.84-1.00)$ & 0.06
\end{tabular}

ppb positive prostate biopsies

a No significant differences were found when considering Gleason $\leq 6$ and Gleason $=7(3+4)$ compared to the rest of Gleason score. Similar lack of significant differences were observed when Gleason $=6$ was compared to Gleason $=7$ or Gleason $=8-10$

patient series, upstaging derived from mpMRI modified RT treatment for three main reasons: alteration of a patient's risk group; extension of target volume due to any type of ECE (T3-T4 patients) in high-risk patients; or treatment with short-term HT due to accumulation of unfavourable factors in intermediate-risk patients. While there are proven benefits in overall survival (OS) among high-risk patients of long-term HT associated with high-dose RT (Zapatero et al. 2015), these benefits are not clear in all intermediate-risk patients (D'Amico 2015). With the available scientific evidence on the matter, many authors recommend associating HT with high-dose RT only in intermediate-risk patients with unfavourable factors (D'Amico 2015), and this is the protocol we have applied at our institution.
Very few studies have evaluated the impact of MRI staging on final decisions in RT treatment (Table 5). We obtained a global alteration of RT treatment in 43.8 or $52.5 \%$ of patients (depending on HT criteria for intermediate-risk patients). Other studies have shown a change in RT treatment of between 8 and $34 \%$. Such variability can be due to several causes: some studies included patients who underwent MRI after starting HT treatment, leading to a reduction of tumour size and consequently a false downstaging (Panje et al. 2015; Chang et al. 2014); on the other hand, most of the studies in this context were performed using 1.5T MRI without endorectal coil (Panje et al. 2015; Yamaguchi et al. 2015; Chang et al. 2014; Jackson et al. 2005) whereas our investigation was conducted using 3T MRI, with higher diagnostic reliability (Park et al. 2007). Morphological MRI has been used in most of the reported series without functional associated studies (Horsley et al. 2015; Yamaguchi et al. 2015; Chang et al. 2014; Jackson et al. 2005). Our study implemented the guide-recommended (Barentsz et al. 2012) morphological T2 sequences along with two functional studies (DWI and DCE), increasing the diagnosis rate, as previously reported (Wu et al. 2012; Verma et al. 2012). In particular, we observed a large number of patients initially categorized as CT1-T2a (239 patients), which might have contributed to such a marked degree of upstaging. On the other hand, in many of the aforementioned studies, HT was administered to all intermediate-risk patients (Panje et al. 2015; Horsley et al. 2015; Yamaguchi et al. 2015; Chang et al. 2014; Jackson et al. 2005), independent of unfavourable risk factors. In this respect, if we had considered administering $\mathrm{HT}$ to all intermediate-risk 
Table 5 Clinical studies evaluating the impact of the staging using MRI in PCa patients treated with RT

\begin{tabular}{|c|c|c|c|c|c|c|c|c|}
\hline Study & Type of MRI & $\mathrm{n}$ & $\begin{array}{l}\text { Field } \\
\text { strength }\end{array}$ & Coil & $\begin{array}{l}\text { Tumor stage } \\
\text { shift (\%) }\end{array}$ & $\begin{array}{l}\text { Risk group } \\
\text { changes ( \%) }\end{array}$ & $\begin{array}{l}\text { Change in RT } \\
\text { (CTV, doses, } \\
\text { HT) (\%) }\end{array}$ & $\begin{array}{l}\text { Technique } \\
\text { validation }\end{array}$ \\
\hline Panje et al. (2015) & Multiparametric & 122 & $1.5 T \& 3 \mathrm{~T}$ & $\mathrm{PAB}$ & 55.7 & 28.7 & 30 & No \\
\hline Horsley et al. (2015) & Morphological & 509 & $1.5 \mathrm{~T}$ & PAB & 20 & 9 & 18 & No \\
\hline $\begin{array}{l}\text { Yamaguchi et al. } \\
\text { (2015) }\end{array}$ & Morphological & 157 & $1.5 \mathrm{~T}$ & PAB & 25 & 9 & $8^{\mathrm{a}}$ & No \\
\hline Couñago et al. (2014) & Multiparametric & 103 & $3 T$ & PAB & 94.1 & 33.9 & 33.9 & Yes \\
\hline Chang et al. (2014) & Morphological & 115 & $1.5 \mathrm{~T}$ & $\mathrm{PAB}$ & 68.6 & 7 & $20^{\mathrm{a}}$ & No \\
\hline Jackson et al. (2005) & Morphological & 199 & $1.5 \mathrm{~T}$ & PAB & 55 & $N R$ & $32.6^{\mathrm{b}}$ & No \\
\hline Present study & Multiparametric & 274 & $3 T$ & $P A B$ & 90.4 & 32.8 & 43.8 or $52.5^{c}$ & Yes \\
\hline
\end{tabular}

Not RT change reported

PAB Phased-array-bodycoil, NR not reported, CTV clinical target volume, HT hormonal therapy

a Exclusive assessment of the CTV change

b Data from T1-T2 to T3-T4 upstaging

c Values according to the HT criteria in intermediate-risk patients

patients, the impact of RT treatment would have decreased to $41.2 \%$. On the other hand, in the literature, conventional clinical staging was performed not only using DRE/TRUS, but also with pelvic CT (Panje et al. 2015), which could have influenced the final results. Lastly, it must be remarked that patients who progressed to the metastatic category due to pelvic node involvement and/or bone metastases were taken into account. In some studies, these patients were excluded from the final analysis (Horsley et al. 2015).

The greatest impact of mpMRI on RT treatment prescription occurs in low-risk or intermediate-risk cT1-T2 patients who progress to $\mathrm{mT} 3-\mathrm{T} 4$, due to the fact that in addition to expanding the target volume to include ECE, we must associate long-term HT with the increased toxicity this entails. Our study shows that $16.7 \%$ of patients were upstaged to T3-T4. This result is similar to that found by Horsley et al.(2015), who investigated the impact on RT treatment prescription of staging using morphological $1.5 \mathrm{~T}$ MRI with body-coil. In their study of 509 patients, Horsley et al. found that $20 \%$ of patients initially categorized as $\mathrm{T} 1-\mathrm{T} 2$ were upstaged to $\mathrm{T} 3-\mathrm{T} 4$ based on MRI findings. On the other hand, Jackson et al. (2005) obtained a $35.2 \%$ change in treatment decision due to MRI findings in T3-T4 patients. This value can be seen as unrealistic, given the fact that in our surgical patient cohort, mpMRI testing showed a low predictive positive value $(58.3 \%)$ for T3 staging; this potentially constitutes an over-diagnosis, and consequent over-treatment and increased toxicity in patients. These data are consistent with a recent meta-analysis that warns of the low sensitivity of MRI in ECE detection (de Rooij et al. 2015). However, a limited number of patients would be under-treated due to the high negative predictive value
(96.2\%) for any ECE detection. In contrast, we obtained $71.0 \%$ for overall accuracy with mpMRI staging, while the value for DRE/TRUS was only $8.8 \%$. Globally, mT staging allowed us to prescribe RT treatments with higher reliability than did cT-staging.

In our series, the global change within the patient risk group was analysed considering the tumour category, as well as the PSA level and the GS. Ours results are comparable to a recent paper published by Panje et al. (32.8 vs $28.7 \%$ ) (Panje et al. 2015). These data suggest that a minimum of approximately $30 \%$ of prostate cancer patients staged with mpMRI could face an alteration of the final RT treatment decision. The ultimate impact on treatment prescription will vary according to the different care protocols in prostate cancer management, such as the criteria to include the seminal vesicles or pelvis within the target volume, administered treatment doses, HT criteria in intermediate-risk patients, and the use of combined brachytherapy with RT. Unfortunately, all these issues are very variable among different institutions (Horsley et al. 2015).

When analysing which patients would benefit most from undergoing MRI for staging purposes, our study, along with those of Panje and Horsley (Panje et al. 2015; Horsley et al. 2015), found significant upstaging in all risk groups-in our case, $86-94 \%$ for any PSA or GS value. In addition, we observed that $66.3 \%$ of low-risk patients progressed to intermediate-risk or high-risk; $18.1 \%$ of intermediate-risk patients progressed to high-risk or metastatic; and $7.7 \%$ of high-risk patients were upstaged to metastatic. Also, of the 45 patients upstaged to T3-T4 because of mT-staging, 11.1, 48.9 and $40.0 \%$ were initially low-risk, intermediate-risk, and high-risk, respectively. Lastly, though there are published studies that 
suggest that tumour detection and location are worse the lower the GS value and tumour size are (Bratan et al. 2013), our univariant and multivariant logistic regression analysis showed no clinical factors associated with lower technique reliability in tumour staging. Consequently, in our clinical environment, all patients with localised prostate cancer who are being considered for RT will benefit from mpMRI staging.

This study presents the following limitations: first, it is a retrospective study with all the inherent limitations associated with this design; second, our follow-up time was not long enough to validate the prognostic significance of upstaging through mpMRI, although this was not the main aim of the study; third, as mpMRI is more accurate than DRE/TRUS and led to a change in T-category or management, this did not lead to increased survival in patients. This hypothesis should be confirmed in a phase three clinical trial; fourth, the radiologist was not blinded, and consequently knew the patients' initial clinical category before the MRI took place. This bias was minimised when quantitative parameters such as ADC or semi-quantitative ones such as time-intensity curves were analysed. In addition, this is common clinical practice and is assumed in most published studies on this matter (Panje et al. 2015; Horsley et al. 2015; Yamaguchi et al. 2015; Chang et al. 2014; Jackson et al. 2005). Lastly, DRE/TRUS reliability was found to be very low in our study (8.8\%), and this could have influenced the elevated upstaging values observed with mpMRI. Having said this, the literature on this subject describes very low reliability for DRE/TRUS in tumour staging $(<50 \%)$, and high interobserver variability (Philip et al. 2005; Smith and Catalona 1995). However, the impact of upstaging on risk-group modification (according to all factors-Tstaging, PSA and GS) was similar to other study using mpMRI (Panje et al. 2015).

In conclusion, mpMRI tumour staging significantly modified the RT treatment decisions in all prostate cancer risk groups. Approximately $30 \%$ of patients may experience a change in their initial risk group based on mpMRI findings. The magnitude of the impact on final RT treatment decisions will depend on the institution's clinical protocol for prostate cancer management.

\footnotetext{
Authors' contributions

FC designed the Project, collected and analyzed data, and drafted the manuscript; EDC, AAD, FJM, MR contributed to project design and revised the drafted manuscript; DSR edited tables and figures and revised the manuscript; IT collected and analyzed data; $\mathrm{KO}$ revised the manuscript; MM revised the manuscript; $\mathrm{LC}$ revised the manuscript. All authors read and approved the final manuscript.

Author details

${ }^{1}$ Department of Radiation Oncology, Hospital Universitario Quiron Madrid, Calle Diego de Velazquez, 1, Pozuelo de Alarcón, 28223 Madrid, Spain.

${ }^{2}$ Department of Radiology, Hospital Universitario Quiron, Madrid, Spain.
}

${ }^{3}$ Clinical Department, School of Biomedical Sciences, Universidad Europea, Madrid, Spain. ${ }^{4}$ School of Doctoral Studies and Research, Universidad Europea, Madrid, Spain. ${ }^{5}$ School of Biomedical Sciences, Universidad Europea, Madrid, Spain. ${ }^{6}$ Hospital Universitario Quiron, Madrid, Spain. ${ }^{7}$ Departament of Radiation Oncology, Hospital Universitario La Princesa, Madrid, Spain.

\section{Acknowledgements}

We wish to thank all the members of the Clinical Radiology, Urology and Pathology Departments as well as MRI technicians of the Hospital Universitario Quiron Madrid for their invaluable collaboration in this study.

\section{Competing interests}

The authors declare that they have no competing interests.

\section{Ethics statement}

All procedures performed in studies involving human participants were in accordance with the ethical standards of the institutional and/or national research committee and with the 1964 Helsinki Declaration and its later amendments or comparable ethical standards.

\section{Informed consent}

For this type of study, formal consent is not required.

Received: 9 October 2015 Accepted: 5 December 2015 Published online: 18 December 2015

\section{References}

AJCC (2010) Cancer staging manual, vol 7th ed. Springer, New York Augustin H, Fritz GA, Ehammer T, Auprich M, Pummer K (2009) Accuracy of 3-Tesla magnetic resonance imaging for the staging of prostate cancer in comparison to the Partin tables. Acta Radiol 50:562-569

Barentsz JO, Richenberg J, Clements R, Choyke P, Verma S, Villeirs G et al (2012) ESUR prostate MR guidelines 2012. Eur Radiol 22:746-757

Boehmer D, Maingon P, Poortmans P, Baron MH, Miralbell R, Remouchamps V et al (2006) Guidelines for primary radiotherapy of patients with prostate cancer. Radiother Oncol 79:259-269

Bratan F, Niaf E, Melodelima C, Chesnais AL, Souchon R, Mège-Lechevallier F et al (2013) Influence of imaging and histological factors on prostate cancer detection and localisation on multiparametric MRI: a prospective study. Eur Radiol 23:2019-2029

Chang JH, Lim Joon D, Nguyen BT, Hiew CY, Esler S, Angus D et al (2014) MRI scans significantly change target coverage decisions in radical radiotherapy for prostate cancer. J Med Imaging Radiat Oncol. 58:237-243

Couñago F, Recio M, Del Cerro E, Cerezo L, Díaz Gavela A, Marcos FJ et al (2014) Role of 3.0 T multiparametric MRI in local staging in prostate cancer and clinical implications for radiation oncology. Clin Transl Oncol 16:993-999

D'Amico AV (2015) Personalizing the duration of androgen-deprivation therapy use in the management of intermediate-risk prostate cancer. J Clin Oncol 33:301-303

de Rooij M, Hamoen EH, Witjes JA, Barentsz JO, Rovers MM (2015) Accuracy of magnetic resonance imaging for local staging of prostate cancer: a diagnostic meta-analysis. Eur Urol. doi:10.1016/j.eururo.2015.07.029

Hayden AJ, Martin JM, Kneebone AB, Lehman M, Wiltshire KL, Skala M et al (2010) Australian \& New Zealand faculty of radiation oncology genitourinary group: 2010 consensus guidelines for definitive external beam radiotherapy for prostate carcinoma. J Med Imaging Radiat Oncol. 54:513-525

Heidenreich A, Bastian PJ, Bellmunt J, Bolla M, Joniau S, van der Kwast T et al (2014) EAU guidelines on prostate cancer. part 1: screening, diagnosis, and local treatment with curative intent-update 2013. Eur Urol 65:124-137

Horsley PJ, Aherne NJ, Edwards GV, Benjamin LC, Wilcox SW, McLachlan CS et al (2015) Planning magnetic resonance imaging for prostate cancer intensity-modulated radiation therapy: impact on target volumes, radiotherapy dose and androgen deprivation administration. Asia Pac J Clin Oncol. 11:15-21

Hricak H, Dooms GC, Jeffrey RB, Avallone A, Jacobs D, Benton WK (1987) Prostatic carcinoma: staging by clinical assessment, $C T$, and MR imaging. Radiology 162:331-336 
Jackson AS, Parker CC, Norman AR, Padhani AR, Huddart RA, Horwich A et al (2005) Tumour staging using magnetic resonance imaging in clinically localised prostate cancer: relationship to biochemical outcome after neo-adjuvant androgen deprivation and radical radiotherapy. Clin Oncol (R Coll Radiol). 17:167-171

Mohler JL, Kantoff PW, Armstrong AJ, Bahnson RR, Cohen M, D'Amico AV et al (2014) Prostate cancer, version 2.2014. J Natl Compr Canc Netw 12:686-718

Mullerad M, Hricak H, Kuroiwa K, Pucar D, Chen HN, Kattan MW (2005) Comparison of endorectal magnetic resonance imaging, guided prostate biopsy and digital rectal examination in the preoperative anatomical localization of prostate cancer. J Urol 174:2158-2163

Panje C, Panje T, Putora PM, Kim SK, Haile S, Aebersold DM et al (2015) Guidance of treatment decisions in risk-adapted primary radiotherapy for prostate cancer using multiparametric magnetic resonance imaging: a single center experience. Radiat Oncol 10:47

Park BK, Kim B, Kim CK, Lee HM, Kwon GY (2007) Comparison of phased-array 3.0-T and endorectal 1.5-T magnetic resonance imaging in the evaluation of local staging accuracy for prostate cancer. J Comput Assist Tomogr 31(4):534-538

Philip J, Dutta Roy S, Ballal M, Foster CS, avlé P (2005) Is a digital rectal examination necessary in the diagnosis and clinical staging of early prostate cancer? BJU Int 95:969-971

Smith DS, Catalona WJ (1995) Interexaminer variability of digital rectal examination in detecting prostate cancer. Urology. 45:70-74
Verma S, Turkbey B, Muradyan N, Rajesh A, Cornud F, Haider MA et al (2012) Overview of dynamic contrast-enhanced MRI in prostate cancer diagnosis and management. AJR Am J Roentgenol 198(6):1277-1288

Wang L, Hricak H, Kattan MW, Chen HN, Kuroiwa K, Eisenberg HF (2007) Prediction of seminal vesicle invasion in prostate cancer: incremental value of adding endorectal MR imaging to the Kattan nomogram. Radiology 242:182-188

Wu LM, Xu JR, Ye YQ, Lu Q, Hu JN (2012) The clinical value of diffusionweighted imaging in combination with T2-weighted imaging in diagnosing prostate carcinoma: a systematic review and meta-analysis. AJR Am J Roentgenol 199:103-110

Yamaguchi S, Ohguri T, Fujii M, Yahara K, Hayashida Y, Fujimoto N et al (2015) Definitive 3D-CRT for clinically localized prostate cancer: modifications of the clinical target volume following a prostate MRI and the clinical benefits. Springerplus. 4:347

Zapatero A, Guerrero A, Maldonado X, Alvarez A, Gonzalez San Segundo C, Cabeza Rodríguez MA et al (2015) High-dose radiotherapy with shortterm or long-term androgen deprivation in localised prostate cancer (DART01/05 GICOR): a randomised, controlled, phase 3 trial. Lancet Oncol. 16:320-327

Zumsteg ZS, Spratt DE, Pei I, Zhang Z, Yamada Y, Kollmeier M et al (2013) A new risk classification system for therapeutic decision making with intermediate-risk prostate cancer patients undergoing dose-escalated external-beam radiation therapy. Eur Urol 64:895-902

\section{Submit your manuscript to a SpringerOpen ${ }^{\circ}$ journal and benefit from:}

- Convenient online submission

- Rigorous peer review

- Immediate publication on acceptance

- Open access: articles freely available online

- High visibility within the field

- Retaining the copyright to your article

Submit your next manuscript at $>$ springeropen.com 